\title{
A Molecular Model of Secondary Battery Cycle-life Pointing Towards a Final Regularity Resembling Carnot-efficiency
}

\author{
Roland H. Pawelke
}

\begin{abstract}
A transition state theory-influenced ideal model of battery cycle-life is outlined and validated by example of the Panasonic NCR18650B Li-battery cell, which shows after 500 cycles a residual capacity of $68.2 \%$ at $100 \%$ depth of discharge (DoD) as by its datasheet. The ideal model, which bases on a minimum failure causality of one reaction event failing per half-cycle, overstates on the logarithmic scale by just $1.9 \%$ for the conditions given, corresponding to an ideal cycle-life of 563 cycles. Generalization of the model towards DoD-ranges yields for the exemplary $(70 ; 30) \%$ and $(100 ; 60) \%$ margins the cycle-life values of 43252 and 23480 , respectively. Because the model relies solely on natural constants, temperature(s) and Crate(s), the conclusion is drawn that a thermodynamic final regularity similar to Carnot-efficiency governs secondary battery cycle-life; this is in contrast to hitherto academic consensus opinion attesting the matter a basically empiric nature.
\end{abstract}

\section{Introduction}

How many charge-discharge cycles a secondary battery may deliver under operational conditions belongs to the prime questions of reversible electrochemical energy storage. The existing models towards that quantity are either of electrochemical-physical character or (semi-)empirical nature: ${ }^{1}$ However, the former are very complex and rely on numerical datasets whose complete availability cannot be taken for granted, the latter are by definition disinterested in causality beyond the evident; yet both approaches emphasize the significance of battery-specific tangibles. While that bias is accountable considering the drive towards actual applications, much less so is the absence of models based on molecular conceptions because the reversible transfer of mass, the one central feature of reversible chemical energy storage, is a matter placed right at the theoretical fundament of physical chemistry: Considering the vast practical significance of the topic, it is not an excessive expectation that at least some kind of ideal idea about the attainable specific energy and battery cycle-life could/should have materialized in the course of the $20^{\text {th }}$ century to the present day.

Yet there is none and it is primarily for that inadequacy of the hitherto literature situation that this author developed an interest in these matters besides touching lines of work: how a superposition of the ideal gas law, classic equilibrium thermodynamics and the structure of the periodic table set the frame to attainable reversible specific energy has already been outlined on previous occasions for two-phase hydrogen storage systems and secondary batteries. ${ }^{2,3}$ However, pinning down in the same spirit how often a reversible reaction may ideally occur under real failure conditions (more relevant for secondary batteries) remained an open issue to the present day. This paper aims for closure on that question and approaches the problem from a transition state theory (TST) angle for TST relates equilibrium thermodynamics to kinetics, thus accounting for an ambiguous role of time.

\section{Methodical Approach}

Central to TST is the concept of a fast upstream equilibrium between the reactants and an activated complex in which the reaction products are virtually already present; the measurable reaction event is triggered by a molecular vibration along the reaction coordinate, resulting in product formation. ${ }^{4}$ This vibration is accounted for by means of a universal frequency factor $k_{\mathrm{B}} T / h$ in which $k_{\mathrm{B}}$ and $h$ 
represent the Boltzmann and the Planck constant, respectively. Equation 1 shows the classic EyringPolanyi equation connecting the reaction rate $k$ to the molar standard Gibbs enthalpy $\Delta G_{\mathrm{m}}{ }^{\circ}$ which governs the equilibrium between reactants and the activated complex.

$k=\frac{k_{\mathrm{B}} T}{h} \mathrm{e}^{-\Delta G_{\mathrm{m}}{ }^{\circ} / R T}$

The basic TST concept of an upstream equilibrium prior the actual reaction event may be applied in adjusted form to secondary batteries because of the fundamental equilibrium nature of reversible (electro-)chemical systems. Hence anything prior the most activated, thus slowest, thus kinetically visible step of the reaction may be subsumed in equilibrium manner, and in a secondary battery the intercalation of the migrating species into the vacant electrode site qualifies as such a bottleneck step (this conception resonates e.g. the principal direction in Li-battery research of aiming at easier intercalation by opening up of the layers of graphite electrodes). Thus, the upstream equilibrium spans the mass transfer from one electrode just upfront the vacant destination site in the other. The shift from a single-phase equilibrium towards a coupled multi-phase one is unproblematic as long as adjustment of the new global equilibrium still occurs faster than the intercalation of the migrating ion into the vacant site. A molecular vibration along the reaction coordinate triggers the apparent reaction event of the charge or discharge process.

However, the present scope requires that said global equilibrium relation is augmented by a specific (i.e. mass) reference: this additional information provides the equilibrium constant with the quality of a reservoir of reaction events because a given amount of system mass can allow for only so many of them. With regard to equation 1 that may be achieved by expressing the reaction rate $k$ via the ratio of specific battery power in $\mathrm{W} \mathrm{kg}^{-1}$ by battery specific energy in $\mathrm{Wh} \mathrm{kg}^{-1}$, illustrating the mass reference does not need to show explicitly. How reaction event count can eventually yield ideal cycle-life is as follows: in each cycle, the real system will invariably experience capacity degradation and the minimum causal increment to that process is one molecular reaction event failing per macroscopic half-cycle respective individual reaction. The loss of one reaction event over one cycle or more does not make sense because failure is a binary matter in this context: by what reason could the chemistry have worked flawlessly in one reaction but then failed in the subsequent one? There is none; in contrast, knowledge of the reason why a reaction event failed is non-essential as it does not matter why the thermodynamic driving force became insufficient, it only matters that it did. By that line of argument, it may be assessed how often the Gibbs enthalpy suffices for moving a secondary battery system across a residual capacity line, with each crossing a reaction event falling away: since the size of the reaction event pool is known, ideal cycle-life becomes determinable.

Hence equation 1 is adjusted towards equations 2 and 3: The mass transfer of the migrating species $M$ between the cathode $C$ and the anode $A$ occurs via two respective global upstream equilibria of the constants $K_{1}$ and $K_{2}$, each reaching from the occupied site by $M$ in one electrode just upfront the vacant destination site in the other. Both equilibria adjust faster than the incorporation of $M$ into the target vacancy. The equilibrium constants are outfitted with an implicit specific reference via the kinetic reaction constants as outlined, providing the connotation of reaction event reservoirs. The reactions occur at two distinct temperatures $T_{1}$ and $T_{2}$. Overall, the respective apparent reaction rates $k_{1}$ and $k_{2}$ are defined in analogy to the classic Eyring-Polanyi equation but the index $\neq$ is omitted because the activated complex is of no concern by actual scope. 


$$
\begin{array}{lll}
\mathrm{C} \cdot \mathrm{M} \rightleftharpoons[\mathrm{C} \cdots \mathrm{M} \cdot \mathrm{A}] \rightarrow \mathrm{A} \cdot \mathrm{M} & \quad-R T_{1} \ln \left(K_{1}\right)=\Delta G_{m, 1}^{\circ}=-\mathrm{R} T_{1} \ln \left(\frac{k_{1} h}{k_{\mathrm{B}} T_{1}}\right) \\
\mathrm{C} \cdot \mathrm{M} \leftarrow[\mathrm{C} \cdot \mathrm{M} \cdot \cdots \mathrm{A}] \rightleftharpoons \mathrm{A} \cdot \mathrm{M} & \Rightarrow & -R T_{2} \ln \left(K_{2}\right)=\Delta G_{\mathrm{m}, 2}^{\circ}=-R T_{2} \ln \left(\frac{k_{2} h}{k_{\mathrm{B}} T_{2}}\right)
\end{array}
$$

\section{Results}

Bringing the macroscopic cycle level and the microscopic reaction event level soundly together is the task at hand: equation 4 displays the base hypothesis of this approach and establishes on basis of the basic thermodynamic equilibrium relation $-R T_{x} \ln \left(K_{x}\right)=\Delta G_{m, x}{ }^{\circ}$ a measure of total reaction event count per macroscopic cycle.

$-\left(\frac{\Delta G_{\mathrm{m}, 1}{ }^{\circ}+\Delta G_{\mathrm{m}, 2}{ }^{\circ}}{R}\right)=T_{1} \ln \left(K_{1}\right)+T_{2} \ln \left(K_{2}\right)=\ln \left(\#_{\text {reaction events }}\right)$

Equation 4 is expressed further in terms of equations 2 and 3 as shown in equation 5.

$-\left(\frac{\Delta G_{\mathrm{m}, 1}^{\circ}+\Delta G_{\mathrm{m}, 2}^{\circ}}{R}\right)=T_{1} \ln \left(\frac{k_{1} h}{k_{\mathrm{B}} T_{1}}\right)+T_{2} \ln \left(\frac{k_{2} h}{k_{\mathrm{B}} T_{2}}\right)=\ln \left(\#_{\text {reaction events }}\right)$

Reversibility implies equal thermodynamic driving forces back and forth and due to that symmetry requirement both Gibbs enthalpies are equal; yet in order to unify indexes, both are expressed by the arithmetic mean $\Delta G_{m, a}{ }^{\circ}$ as equation 6 shows.

$-\left(\frac{\Delta G_{\mathrm{m}, 1}^{\circ}+\Delta G_{\mathrm{m}, 2}^{\circ}}{R}\right)=-\left(\frac{2 \Delta G_{\mathrm{m}, \mathrm{a}}^{\circ}}{R}\right)=\left(T_{1} \ln \left(\frac{k_{1} h}{k_{\mathrm{B}} T_{1}}\right)+T_{2} \ln \left(\frac{k_{2} h}{k_{\mathrm{B}} T_{2}}\right)\right)$

The pre-logarithmic temperatures $T_{1}$ and $T_{2}$ are expressed in terms of their arithmetic mean $T_{\mathrm{a}}$ accordingly, which is isolated and brought to the left side as equations 7 a to $7 \mathrm{c}$ show.

$$
\begin{aligned}
& -\left(\frac{2 \Delta G_{\mathrm{m}, \mathrm{a}}^{\circ}}{R}\right)=\left(\left(2 T_{\mathrm{a}}-T_{2}\right) \ln \left(\frac{k_{1} h}{k_{\mathrm{B}} T_{1}}\right)+\left(2 T_{\mathrm{a}}-T_{1}\right) \ln \left(\frac{k_{2} h}{k_{\mathrm{B}} T_{2}}\right)\right) \\
& -\left(\frac{2 \Delta G_{\mathrm{m}, \mathrm{a}}}{R T_{\mathrm{a}}}\right)=\left(2-\frac{T_{2}}{T_{\mathrm{a}}}\right) \ln \left(\frac{k_{1} h}{k_{\mathrm{B}} T_{1}}\right)+\left(2-\frac{T_{1}}{T_{\mathrm{a}}}\right) \ln \left(\frac{k_{2} h}{k_{\mathrm{B}} T_{2}}\right)
\end{aligned}
$$


$-\left(\frac{2 \Delta G_{\mathrm{m}, \mathrm{a}}{ }^{\circ}}{R T_{\mathrm{a}}}\right)=\left(2-\frac{2 T_{2}}{T_{1}+T_{2}}\right) \ln \left(\frac{k_{1} h}{k_{\mathrm{B}} T_{1}}\right)+\left(2-\frac{2 T_{1}}{T_{1}+T_{2}}\right) \ln \left(\frac{k_{2} h}{k_{\mathrm{B}} T_{2}}\right)$

The operations to this point do not account for the transitional, cyclic nature of the issue and this feature is now accounted for by adjusting both In-terms towards geometric mean temperature $T_{\mathrm{g}}$ via the basic thermodynamic equilibrium relation $-R T_{x} \ln \left(K_{x}\right)=\Delta G_{\mathrm{m}, x}{ }^{\circ}$ as equations $8 \mathrm{a}$ and $8 \mathrm{~b}$ show.

$$
\begin{aligned}
& -\left(\frac{\Delta G_{\mathrm{m}, x} x^{\circ}}{R}\right)=T_{x} \ln \left(k_{x}\right)=T_{x} \ln \left(\frac{k_{x} h}{k_{\mathrm{B}} T_{\mathrm{x}}}\right) \Rightarrow T_{x} \ln \left(\frac{k_{x} h}{k_{\mathrm{B}} T_{x}}\right)=T_{\mathrm{g}} \ln \left(\frac{k_{g} h}{k_{\mathrm{B}} T_{\mathrm{g}}}\right) \Rightarrow \ln \left(\frac{k_{x} h}{k_{\mathrm{B}} T_{x}}\right)=\frac{T_{\mathrm{g}}}{T_{x}} \ln \left(\frac{k_{\mathrm{g}} h}{k_{\mathrm{B}} T_{\mathrm{g}}}\right) \\
& -\left(\frac{2 \Delta G_{\mathrm{m}, \mathrm{a}}{ }^{\circ}}{R T_{\mathrm{a}}}\right)=\ln \left(\frac{k_{\mathrm{g}} h}{k_{\mathrm{B}} T_{\mathrm{g}}}\right)\left(\left(2-\frac{2 T_{2}}{T_{1}+T_{2}}\right) \frac{T_{\mathrm{g}}}{T_{1}}+\left(2-\frac{2 T_{1}}{T_{1}+T_{2}}\right) \frac{T_{\mathrm{g}}}{T_{2}}\right)=\ln \left(\#_{\text {reaction events }}\right)
\end{aligned}
$$

The temperature-related terms on the right of equation 8 a can be much simplified and the geometric mean reaction constant $k_{\mathrm{g}}$ in $\mathrm{s}^{-1}$ may be expressed in terms of both $C$-rates (commonly in $\mathrm{h}^{-1}$ ) under the premise of constant voltage, as equation 9a shows. Equation $9 \mathrm{~b}$ displays the transition from reaction events to cycles, dividing equation 9a by two.

$$
\begin{aligned}
& -\left(\frac{2 \Delta G_{\mathrm{m}, \mathrm{a}}{ }^{\circ}}{R T_{\mathrm{a}}}\right)=\frac{2 T_{\mathrm{g}}}{T_{\mathrm{a}}} \ln \left(\frac{\sqrt{C-\text { rate }_{\text {charge }} \cdot C \text {-rate }} \text { discharge }}{3600} \frac{h}{k_{\mathrm{B}} T_{\mathrm{g}}}\right)=\ln \left(\#_{\text {reaction events }}\right) \\
& -\left(\frac{\Delta G_{\mathrm{m}, \mathrm{a}}^{\circ}}{R T_{\mathrm{a}}}\right)=\frac{T_{\mathrm{g}}}{T_{\mathrm{a}}} \ln \left(\frac{\sqrt{C \text {-rate }} \text { charge }_{\text {ch }} \cdot \text {-rate }_{\text {discharge }}}{3600} \frac{h}{k_{\mathrm{B}} T_{\mathrm{g}}}\right)=\ln \left(\#_{\text {cycles }}\right)
\end{aligned}
$$

How may a change $\Delta$ to the thermodynamic reaction quantities on the left respective middle of equation $9 \mathrm{~b}$ affect cycle number on the right? This question vital to the issue at hand is expressed in equation 10, abbreviating the argument of the Planck-Boltzmann In-term as quantity $A$ and the prelogarithmic temperature quotient as variable $\alpha$.

$$
\begin{aligned}
& -\left(\frac{\Delta \Delta G_{\mathrm{m}, \mathrm{a}}{ }^{\circ}}{R T_{\mathrm{a}}}\right)=\Delta \ln (A)^{\alpha}=\ln \left(\Delta \#_{\text {cycles }}\right) \quad ? \\
& A=\left(\frac{\sqrt{C \text {-rate }_{\text {charge }} \cdot C \text {-rate }} \text { discharge }_{2}}{3600} \frac{h}{k_{\mathrm{B}} T_{\mathrm{g}}}\right) \quad \alpha=\frac{T_{\mathrm{g}}}{T_{\mathrm{a}}}
\end{aligned}
$$

The middle-right section of equation 10 is taken and solved for $\ln \left(\#_{\text {cycles }}\right)$, equations $11 \mathrm{a}$ and $11 \mathrm{~b}$ show accordingly.

$$
\Delta \ln (A)^{\alpha}=\ln \left(\#_{\text {cycles }}\right)+\ln (\Delta)
$$


$-\Delta \ln \left(\frac{1}{A}\right)^{\alpha}+\ln \left(\frac{1}{\Delta}\right)=\ln \left(\#_{\text {cycles }}\right)$

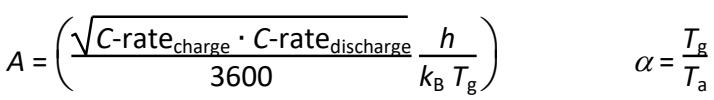

The seemingly sole systematic way towards figuring the relation between changes $\Delta$ is a quadratic approach because only for this case clear boundary conditions can be established: equation 12 shows how equation $11 \mathrm{~b}$ relates by principle to the vertex form of a quadratic equation.

$\begin{array}{ll}-\Delta \ln \left(\frac{1}{A}\right)^{\alpha}+\ln \left(\frac{1}{\Delta}\right) & =\ln \left(\#_{\text {cycles }}\right) \\ -x^{2} a+b+b & =\mathrm{f}(x)\end{array}$

In equation 12 , variable $a$ respective $\ln (1 / A)^{\alpha}$ determines the jaw width of this downward open parabola and since its vertex has to be at $50 \%$ capacity, $(x-0.5)^{2}$ applies instead of $x^{2}$. However, this parabola has only one zero-crossing in the vertex but the boundary conditions demand two zerocrossings at 0 and 1 (the margins of relative capacity): this requires a shift above the $x$-axis by $\ln (1 / \Delta)$ $=1 / 4 \ln (1 / A)^{\alpha}$ and hence, a quarter of the jaw width value gives the apex value of cycle-life, equation 13 shows this accordingly.

$$
\begin{aligned}
& -(x-0.5)^{2} \ln \left(\frac{1}{A}\right)^{\alpha}+\frac{1}{4} \ln \left(\frac{1}{A}\right)^{\alpha}=\ln \left(\#_{\text {cycles }}\right) \quad \Rightarrow \quad \frac{1}{4} \ln \left(\frac{1}{A}\right)^{\alpha}=\ln \left(\#_{\text {cycles }}\right)_{c_{50}} \\
& A=\left(\frac{\sqrt{C \text {-rate }_{\text {charge }} \cdot C \text {-rate }_{\text {discharge }}}}{3600} \frac{h}{k_{\mathrm{B}} T_{\mathrm{g}}}\right) \quad \alpha=\frac{T_{g}}{T_{\mathrm{a}}}
\end{aligned}
$$

For the sake of clarity, the line of argument up to equation 13 is summarized in three points: first, because the minimum failure causality warrants the loss of two reaction events per cycle, the cyclelife value is basically limited to the number reaction events of a half-cycle respective single reaction (equation $9 \mathrm{~b}$ ). Second, the equilibrium balance point of the system lies at $50 \%$ of possible reaction events. Third, fixing that equilibrium balance point in the vertex of a downward open parabola, while accounting for zero-crossings at interval borders 0 and 1, entails that the $25 \%$ fraction of the total reaction event pool by value equals maximum ideal cycle-life.

This actually explains why a deep discharge annuls the reversible character of a secondary battery so speedily since violating the fundamental premise of reversibility: For example, it is well-known that discharging a secondary Li-battery beyond $50 \%$ residual Li-content in the exemplary graphite anode, thus $<\mathrm{C}_{6} \mathrm{Li}_{0.5}$, is very disadvantageous to cycle-life and $100 \%$ DoD mean reaction event-wise that actually only $50 \%$ of the total lithium is de-intercalated. That seemingly empiric finding is sound in terms of the reaction event conception since the equilibrium balance point lies at $50 \%$ of possible reaction events, representing for symmetry reasons the baseline to reversibility. Because two reaction events are lost per cycle at minimum, half the reaction events of that balance point at $50 \%$ figure the maximum ideal cycle-life value at $100 \%$ DoD, which equation 14 shows. 
$\frac{1}{4} \ln \left(\frac{1}{A}\right)^{\alpha}=\ln \left(\#_{\text {cycles }}\right) c_{50}=\ln \left(\#_{\text {cycles, } \max }\right)_{\mathrm{DoD}(1 \leftrightarrow 0)}$

However, the actual distribution function of cycle-life has not parabola shape but those of a negated e-function, which may be fitted on basis of equation 14, though. Equation 15a gives an impression of how that might be achieved in a straightforward way, with $\beta=1 / 4 \ln (1 / A)^{\alpha}$, but it is not fully compliant with interval boundary requirements as placing the intercept by 1 unit too low. Thus, fitting equation 15 a properly into interval bounds requires further adjustment and equation $15 \mathrm{~b}$ shows the simplest possible way towards that end (for a comparative plot of equations $13,15 \mathrm{a}$ and $15 \mathrm{~b}$ please refer to the discussion section respective the electronic supplementary information).

$$
\begin{aligned}
& -\beta \mathrm{e}^{(x-1) \ln (\beta))}+\beta=\mathrm{f}(x) \quad \mid \quad \beta=\frac{1}{4} \ln \left(\frac{1}{A}\right)^{\alpha} \\
& \ln \left(\#_{\text {cycles, } \max }\right)_{\operatorname{DoD}(1 \leftrightarrow 0)}=-\beta x \mathrm{e}^{(x-1) \ln (\beta))}+\beta
\end{aligned}
$$

Equation $15 \mathrm{~b}$ gives the cycle-life at $100 \%$ DoD until a residual capacity $x$ is reached: it may be via term $\beta$ generalized towards any $(y ; z)$-DoD margin because the resizing of the base variable $\beta$ on the logarithmic scale requires merely the addition of a In-value of a term accounting for the effect of the shifted DoD-limits $(y ; z)$ with $1 \geq y>z \geq 0$. Yet the premises apply that said term must be quadratic by nature and vanish for $100 \% \operatorname{DoD}(y=1 ; z=0)$ : equation 16 shows how this scale factor termed $\gamma$ may be expressed accordingly, it is noteworthy that variables $y$ and $z$ refer to the DoD scale while variable $x$ relates to the capacity/reaction event scale and that $100 \%$ DOD equal $50 \%$ of capacity in terms of reaction events.

$$
\frac{1}{4} \ln \left(\frac{1}{A}\right)^{\alpha}+\ln (\gamma)=\ln \left(\#_{\text {cycles, } \max }\right)_{\operatorname{DoD}(y \leftrightarrow z)} \quad \mid \quad \gamma=\left(\frac{(z-0.5)^{2}}{(y-0.5)^{2}}+\frac{z^{2}}{y^{2}}\right) ; 1 \geq y>z \geq 0
$$

The quotient of the squared distances of DoD-boundaries from the equilibrium balance point in the argument of $\ln (\gamma)$ is fix but there is the principal possibility of a further factor to the other term as that one will vanish for $z=0$ in any case. Because quantity $\gamma$ represents the squared radius of a unity circle in Cartesian coordinates, the area ratio $z^{2} / y^{2}$ is multiplied by an e-function with the reciprocal $3^{\text {rd }}$ binomial formula $1^{2} /\left(y^{2}-z^{2}\right)$ as exponent for scale in proportion to the baseline unity circle at $100 \%$ DoD. Equation 16 thus completed yields equation 17.

$$
\ln \left(\#_{\text {cycles, } \max }\right)_{\operatorname{DoD}(y \leftrightarrow z)}=\frac{1}{4} \ln \left(\frac{1}{A}\right)^{\alpha}+\ln \left(\frac{(z-0.5)^{2}}{(y-0.5)^{2}}+\frac{z^{2}}{y^{2}} \mathrm{e}^{1 /\left(y^{2}-z^{2}\right)}\right) \quad \mid \quad \operatorname{DoD}(1 \geq y>z \geq 0)
$$


Equation 17 gives the ideal cycle-life at any DoD $(y ; z)$-margin, allowing in concert with equation $15 b$ the complete cycle-life assessment of any secondary battery system.

\section{Discussion}

It is straightforward to validate equations $15 \mathrm{~b}$ and 17 by means of an example and the Panasonic NCR18650B Li-ion cell is an ideal item towards that end for it represents an iconic reference in terms of specific energy and the high interest vested in secondary Li-battery cycle-life: The cycle-life curve presented in its datasheet refers to $100 \% \mathrm{DoD}$ at $298.15 \mathrm{~K}$ and $\mathrm{C}$-rates of 0.5 and 1 respectively; the exemplary cell starts just above $3300 \mathrm{mAh}$ initial capacity and shows a decrease to $2250 \mathrm{mAh}(68.2$ $\%)$ after 500 cycles. $^{5}$ These base parameters yield by equation $9 \mathrm{~b}$ the value of $\ln (1 / A)^{\alpha}=37.9928 \approx$ $38.0(\alpha=1)$, a quarter of which gives the $\beta$-value of 9.5. Placing that into equation $15 \mathrm{~b}$ yields for $x=$ 0.682 a cycle-life In-value of 6.3334 which is just $1.9 \%$ above $\ln (500)=6.2146$ : the difference between ideal and real cycle life is 63 cycles or $+12.6 \%$, a remarkable agreement considering the minimum failure causality underlying equation $15 \mathrm{~b}$ which gives an idea about the magnitude of the real degradation processes. A comparative plot of equations $13,15 \mathrm{a}$ and $15 \mathrm{~b}$ is available as electronic supplementary information.

Applying these data further to equation 17 for the ideal cycle-life at DoD-margins, e.g. (70; 30) \% and $(100 ; 60) \%$, changes the value of $\beta=9.5$ by +1.1748 and +0.5639 , respectively: This equals cycle-life numbers of 43252 and 23480, reflecting the well-known empiric fact that DoD-margin bounds exert a comparably much stronger impact on cycle-life than the fraction of capacity fenced-in. Adjustment of the model towards more pronounced failure causality is possible at little effort.

The findings of this work suggest nothing less than a revision of the hitherto academic consensus on the subject, attesting secondary battery cycle-life a basically empiric nature. That is not a very sound ascertainment but accountable considering that the multi-layered, integrated complex structure of the problem at hand prevents its breaking down in the usual manner of technical problem-solving. However, since that premise of authorative opinion is diametrically opposed to those underlying this work, discussing outcomes in its terms is fairly pointless and the only common ground might be at the factual level of cycle-life figures. Yet the notion of secondary battery cycle-life being a matter of empiric nature is defective from the start and actually, reversible chemical systems could not be any farther away from qualifying as purely empiric entities, alone for the reason that the thermodynamic conception of reversibility presupposes ideal behaviour. In line with that fact, this work arrives at the conclusion that a final regularity similar to Carnot-efficiency applies to secondary battery cycle-life as it can be defined on sole basis of temperature level(s), $C$-rate(s) and natural constants.

\section{Conclusion}

Approaching the subject of secondary battery cycle-life from a physical chemistry fundamentals angle leads to an ideal model of general validity. The result suggests further the central significance of a thermodynamic final regularity similar to Carnot-efficiency, which is in contrast to the hitherto academic consensus opinion, attesting the matter a basically empiric nature. 


\section{Acknowledgements}

The idea for this paper spun-off from the author's prime line of work done at FOTEC Forschungs- und Technologietransfer GmbH under European Space Agency grant 4000105330/12/NL/CLP and European Defence Agency grant A-1341-RT-GP which is all gratefully acknowledged.

\section{Conflicts of Interest}

No conflicts to declare.

\section{References}

(1) Motapon, S. N.; Lachance, E.; Dessaint, L.-A.; Al-Haddad, K. A Generic Cycle Life Model for Lithium-Ion Batteries Based on Fatigue Theory and Equivalent Cycle Counting. IEEE Open J. Ind. Electron. Soc. 2020, 1, $207-217$. https://doi.org/10.1109/OJIES.2020.3015396.

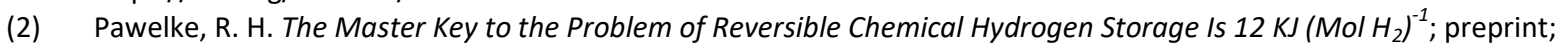
2021. https://doi.org/10.26434/chemrxiv.6940379.v11.

(3) Pawelke, R. H. The Straightforward Route Towards the Theoretical Specific Free Enthalpy of Electrochemical Reactions; preprint; 2021. https://doi.org/10.26434/chemrxiv.8799188.v3.

(4) Ptáček, P.; Šoukal, F.; Opravil, T. Introduction to the Transition State Theory. In Introducing the Effective Mass of Activated Complex and the Discussion on the Wave Function of this Instanton; Ptáček, P., Opravil, T., Šoukal, F., Eds.; InTech, 2018. https://doi.org/10.5772/intechopen.78705.

(5) Perez, A.; Quintero, V.; Rozas, H.; Jaramillo, F.; Moreno, R.; Orchard, M. Modelling the Degradation Process of Lithium-Ion Batteries When Operating at Erratic State-of-Charge Swing Ranges. In 2017 4th International Conference on Control, Decision and Information Technologies (CoDIT); IEEE: Barcelona, 2017; pp 0860-0865.

https://doi.org/10.1109/CoDIT.2017.8102703. 